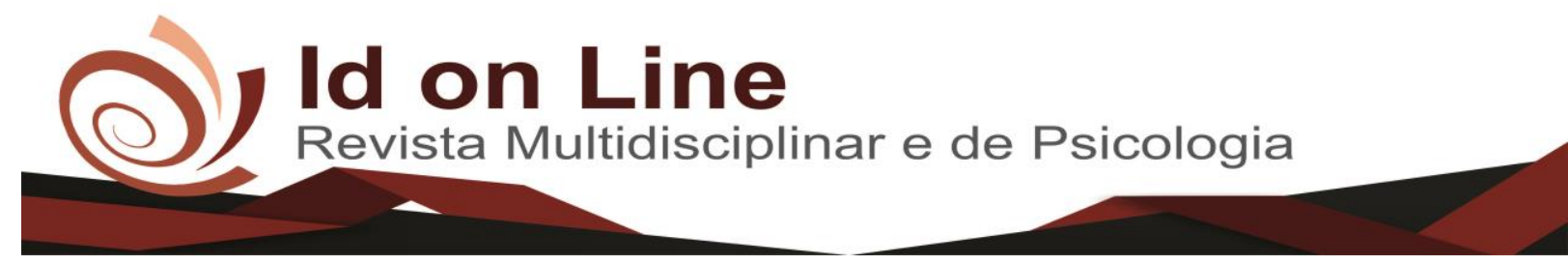

Artigo

\title{
Sintomas do Vaginismo em Mulheres Submetidas à Episiotomia
}

\author{
Andressa Moura Alves ${ }^{1}$; Rosana Porto Cirqueira ${ }^{2}$
}

\begin{abstract}
Resumo: O vaginismo é uma contração vaginal de forma involuntária, gerando muitas vezes no ato da relação sexual espasmo dos músculos da pelve, que acometem as mulheres, causando grande desconforto, dor, ardência durante a penetração e incapacidade total de ter relação sexual. A episiotomia é um método cirúrgico realizado com muita frequência durante o parto normal, para ajudar na saída da criança. $\mathrm{O}$ estudo analisado tem como objetivo geral verificar a presença dos sintomas do vaginismo em mulheres submetidas à episiotomia. A pesquisa trata-se de um estudo descritivo com delineamento transversal, com abordagem quantitativa, onde participaram do estudo pacientes com sintomas de vaginismo que foram submetidas à episiotomia, de forma voluntária, por conveniências, desde que preencham os critérios de inclusão e exclusão. Será aplicado o questionário de autoria própria para colher o perfil sociodemográfico e obstétrico, o outro questionário a ser aplicado é o International Consultation on Incontinence Questionnaire - Short Form (ICIQ-SF) que é um questionário simples, breve e auto administrável, escolhido para ser traduzido e adaptado para a cultura brasileira por avaliar rapidamente o impacto da incontinência urinária (IU) na qualidade de vida e qualificar a perda urinária de pacientes de ambos os sexos, e o Female Sexual Function Index (FSFI) que é um questionário breve, que pode ser auto aplicado, e que se propõe avaliar a resposta sexual feminina em seis domínios: desejo sexual, excitação sexual, lubrificação vaginal, orgasmo, satisfação sexual e dor. Será incluído no projeto indivíduos com idade acima ou igual á 18 anos. Esperase, ao final deste estudo, contribuir para novos caminhos a serem explorados.
\end{abstract}

Palavras-chave: Vaginismo. Episiotomia. Questionário.

\section{Symptoms of Vaginismus in Women with Episiotomy}

\begin{abstract}
Vaginismus is an involuntary vaginal contraction, often generating in the act of intercourse spasm of the pelvic muscles, which affect women, causing great discomfort, pain, burning during penetration and total inability to have sexual intercourse. Episiotomy is a surgical method performed very often during normal delivery to help with the delivery of the child. The objective of this study was to verify the presence of vaginismus symptoms in women submitted to episiotomy. The research is a descriptive study with a cross-sectional, quantitative approach, in which patients with symptoms of vaginismus who underwent episiotomy were voluntarily treated for convenience provided that they met the inclusion and exclusion criteria. The selfadministered questionnaire will be applied to collect the sociodemographic and obstetric profile, the other questionnaire to be applied is the International Consultation on Incontinence Questionnaire - Short Form (ICIQSF), which is a simple, brief and self-administered questionnaire chosen to be translated and adapted to Brazilian culture for rapid evaluation the impact of urinary incontinence (IU) on quality of life and qualify the urinary loss of patients of both sexes, and Female Sexual Function Index (FSFI), which is a brief questionnaire that can be selfapplied and which proposes to evaluate the female sexual response in six domains: sexual desire, sexual arousal, vaginal lubrication, orgasm, sexual satisfaction and pain. Individuals aged 18 or over will be included in the project. It is hoped, at the end of this study, to contribute to new paths to be explored.
\end{abstract}

Key words: Vaginismus. Episiotomy. Quiz.

\footnotetext{
${ }^{1}$ Faculdade Independente do Nordeste , Brasil. Contato: andressa.fisio@live.com;

${ }^{2}$ Especialização em Fisioterapia em Terapia Manual e Postural pelo Centro Universitário de Maringá, Brasil. Docente da Faculdade Independente do Nordeste, Brasil.
} 


\section{Introdução}

O vaginismo é uma contração vaginal de forma involuntária, gerando muitas vezes no ato da relação sexual espasmo dos músculos da pelve, que acometem as mulheres, causando grande desconforto, dor, ardência durante a penetração e incapacidade total de ter relação sexual, onde o mesmo é a segunda causa de transtorno na disfunção sexual, sendo, estimada a um risco de 8,5 vezes maior para a disfunção no pós-parto. Perante as investigações com as puérperas, as causas do vaginismo são as mesmas da dispaurenia, mas que não tem bastante conhecimento em relação com o próprio corpo delas e das manifestações que aparecem à frente de confundir a dor, a dificuldade ou a impossibilidade ao coito (HOLANDA, et al., 2014).

No Brasil aproximadamente cerca de 2 mulheres entre 1000 têm vaginismo, no entanto, esse número pode ser muito mais alto, mas, é difícil dizer os dados estatísticos sobre essa condição devido à causa de muitas mulheres que tem vergonha e constrangimento que são intimidadas por esse tabu, e não procuram tratamento, atuando no período normal da vida de uma mulher quando ela tenta intercurso sexual pela primeira vez (DE LIMA et al., 2018).

As disfunções da Musculatura do assoalho pélvico (MAP) como hipertonia são consideradas no crescimento do vaginismo. Mas, ainda não está definido se esses distúrbios dos MAP são predisponentes ou se caracterizam como um sintoma. História de contaminações comuns que levam as pacientes a sentirem dores ou irritação durante o ato sexual, como a dispareunia, pode evoluir para o vaginismo. Bem como a rejeição do homossexualismo pelas mulheres, que tentam a relação com pessoas do sexo oposto mesmo não sentindo desejo pelo parceiro, e o fato de trocar esse parceiro sexual pode resultar na ausência dessa alteração. Uma fase frequente desse problema é o período da menopausa devido à atrofia genital (PEREIRA, 2015).

A episiotomia é um método cirúrgico realizado com muita frequência durante o parto normal, para ajudar na saída da criança. Procedimento de uso liberal, mas, que se trata de uma conduta muito frequente que é utilizada de maneira inadequada. É aplicada anestesia para que a mulher não sinta nenhum tipo de dor durante o trabalho de parto, mas, em geral, nas primeiras 2 e 3 semanas após o parto é comum sentir dor e desconforto ao redor da episiotomia, esta indicada com o aumento de fator de risco de choque, inflamação, dor e é considerada um dos únicos procedimentos que é realizado sem qualquer consentimento do paciente e sem que ela seja informada sobre suas indicações e contraindicações (CAMBOIM et al., 2017). 
A Organização Mundial de Saúde (OMS) apresenta que não existem evidências garantidas e que o uso liberal ou rotineiro da episiotomia possua um efeito benéfico, mas há indícios claros de que pode provocar dano, sugere uma taxa ideal de episiotomia nos variados serviços em torno de $10 \%$, realidade em muitos países europeus. Lamentavelmente, no Brasil, em uns centros, a condição é ainda contrária, pois o procedimento é realizado em, aproximadamente, $94 \%$ dos partos vaginais. Apesar das atuais referências sobre a realização da episiotomia seletiva, a frequência segue bastante elevada (DE LIMA et al., 2018).

Diante deste contexto, torna-se importante a realização deste estudo, uma vez que a presença do vaginismo em mulheres que foram submetidas à episiotomia, pode afetar a qualidade de vida dessas mulheres.

Desta forma, o estudo pode contribuir no direcionamento do prognóstico dentro da área de fisioterapia, buscando também a prevenção de mais agravos decorrentes destes sintomas.

$\mathrm{O}$ estudo analisado tem como objetivo geral verificar a presença dos sintomas do vaginismo em mulheres submetidas à episiotomia.

\section{Materiais e Métodos}

Trata-se de um estudo descritivo com delineamento transversal, com abordagem quantitativa, sendo do tipo probabilístico. A pesquisa foi realizada em uma Unidade de Saúde situada no sudoeste baiano, localizada no município de Vitória da Conquista - BA. Dezesseis sujeitos participaram da pesquisa, sendo divididas de forma igual entre os grupos.

Foi aplicado um questionário de autoria própria para colher perfil sociodemográfico e obstétrico, contendo: dados pessoais dos pacientes, como: nome, telefone pra contato, endereço, idade, sexo, cor, estado civil, profissão, escolaridade, tabagismo, obesidade, sedentarismo, sintomas, tipo, posição e complicação no parto, realização da episiotomia.

Para se alcançar os objetivos propostos por este estudo, foi aplicado o ICIQ-SF que é um questionário simples, rápido e auto administrável, escolhido para ser traduzido e adaptado para a cultura brasileira por avaliar rapidamente o impacto da incontinência urinária na qualidade de vida e qualificar a perda urinária de pacientes de ambos os sexos. É constituído de quatro questões que avaliam a frequência, a gravidade e o impacto da IU, além da soma de oito itens de auto diagnóstico, relacionados às causas ou a situações de IU sofridas pelos pacientes. Foi completamente desenvolvido e validado na língua inglesa. Apresenta um escore final de 0 a 100, no qual zero corresponde a pior estado geral de saúde e 100 a melhor estado de saúde. 
O FSFI é um questionário rápido, que consegue ser auto aplicado, e que se pretende avaliar a resposta sexual feminina em seis domínios: desejo sexual, excitação sexual, lubrificação vaginal, orgasmo, satisfação sexual e dor. Para isso, apresenta 19 questões que analisam a função sexual nas últimas quatro semanas. Para cada pergunta existe um padrão de resposta cujas opções recebem pontuação de 0 a 5 de maneira crescente em relação à presença da função questionada. Apenas nas perguntas sobre dor a pontuação é definida de forma invertida. Um escore total é apresentado ao final da aplicação, decorrência da soma dos resultados de cada domínio multiplicada por um fator que assemelha a influência de cada domínio no escore total.

Muito embora o instrumento não tenha a competência de distinguir a etapa da resposta modificada, a partir de um ponto de corte do escore total definido como 26 pontos seria possível discriminar entre as amostras com maior e menor perigo de apresentar disfunção sexual, demostrando que valores idênticos ou abaixo desse ponto indicariam disfunção sexual. É nesse modo que o FSFI agrega as qualidades de ser prático para utilização em estudos populacionais, modificar medidas subjetivas em materiais objetivos, quantificáveis e analisáveis e quantificar a força relativa de cada domínio ou parte da resposta sexual feminina. Outros benefícios desse questionário residem no fato de ser um índice bem entendido, validado e com técnica de analisar intervenções terapêuticas.

O estudo iniciou após a aprovação pelo Comitê de Ética da Instituição sob o número do parecer: 2.883.675 e após todas as pacientes assinarem o termo de consentimento livre e esclarecido (TCLE). A análise dos dados se deu da seguinte forma: Anormalidade da amostra foi avaliada pelo teste de Shapiro-Wilk, foi adotado o nível de $\mathrm{p}<0,05$ para a significância estatística. Para avaliação dos dados foi utilizado pacote estatístico SPSS versão 22.0 for windows.

\section{Resultados}

A média da idade do grupo 1 que realizou episiotomia foi de $31,38 \pm 7,78$, enquanto do grupo 2, que é das mulheres que não realizaram episiotomia, foi de $24,88 \pm 2,8$. A idade possui correlação estatística significante entre os grupos $(p=0,042)$, o que nos faz inferir que mulheres que realizaram episiotomia foram mais velhas. Os outros doze indivíduos foram eliminados devido a problemas período puerperal e/ou não disponibilidade de tempo, critérios de exclusão 
deste trabalho. As pacientes foram abordadas pela acadêmica para preencher os questionários com a duração de 20 minutos.

O estado civil predominante para ambos os grupos foi solteiro G1 50,0\%; G2 75,0\%. O nível de escolaridade para o G1 de ensino médio incompleto $(50,0 \%)$ e para o G2 ensino superior incompleto $(37,5 \%)$, cor parda predominantemente para os dois grupos G1 $(62,5 \%)$ e G2 (75,0\%), com ausência de obesidade em ambos os grupos (87,5\%), e não foi verificada diferenças nos percentuais para sedentarismo e atividade física (Tabela 1).

Tabela 1. Características biosociodemográficas da amostra. Vitoria da Conquista - BA, 2018

\begin{tabular}{|c|c|c|c|}
\hline Características & $\mathbf{G 1}^{1}(\mathrm{n}=8)$ & $\mathbf{G 2}^{2}(\mathrm{n}=8)$ & $p^{*}$ \\
\hline Idade, anos $\left(M^{3} \pm D P^{4}\right)$ & $31,38 \pm 7,78$ & $24,88 \pm 2,8$ & 0,042 \\
\hline \multicolumn{3}{|l|}{ Estado civil, $n(\%)$} & $p^{* *}$ \\
\hline Solteira & $4(50,0)$ & $6(75,0)$ & \multirow{4}{*}{0,523} \\
\hline Casada & $2(25,0)$ & $2(25,0)$ & \\
\hline Divorciada & $1(12,5)$ & - & \\
\hline União estável & $1(12,5)$ & - & \\
\hline \multicolumn{4}{|l|}{ Escolaridade, $n(\%)$} \\
\hline Ensino fundamental completo & $1(12,5)$ & - & \multirow{5}{*}{0,189} \\
\hline Ensino médio completo & $2(25,0)$ & - & \\
\hline Ensino médio incompleto & $4(50,0)$ & $3(37,5)$ & \\
\hline Ensino superior completo & - & $2(25,0)$ & \\
\hline Ensino superior incompleto & $1(12,5)$ & $3(37,5)$ & \\
\hline \multicolumn{4}{|l|}{ Cor, $n(\%)$} \\
\hline Branca & $2(25,0)$ & $1(12,5)$ & \multirow{4}{*}{0,489} \\
\hline Amarela & - & $1(12,5)$ & \\
\hline Parda & $5(62,5)$ & $6(75,0)$ & \\
\hline Negra & $1(12,5)$ & - & \\
\hline \multicolumn{4}{|l|}{ Obesidade, $n(\%)$} \\
\hline Sim & $1(12,5)$ & $1(12,5)$ & \multirow{2}{*}{0,767} \\
\hline Não & $7(87,5)$ & $7(87,5)$ & \\
\hline \multicolumn{4}{|l|}{ Sedentarismo, $n(\%)$} \\
\hline Sim & $4(50,0)$ & $4(50,0)$ & \multirow{2}{*}{0,689} \\
\hline Não & $4(50,0)$ & $4(50,0)$ & \\
\hline \multicolumn{4}{|l|}{ Atividade física, $n(\%)$} \\
\hline Sim & $4(50,0)$ & $4(50,0)$ & \multirow{2}{*}{0,689} \\
\hline Não & $4(50,0)$ & $4(50,0)$ & \\
\hline
\end{tabular}

${ }^{1}$ Grupo Episiotomia ; ${ }^{2}$ Grupo Ausência de Episiotomia; ${ }^{3}$ Média; ${ }^{4}$ Desvio padrão amostral; ${ }^{*}$ Teste t-Student independente; **Teste qui-quadrado de Pearson. Fonte: Dados da pesquisa. 
O tipo de parto predominantemente no grupo 1 foi o vaginal com $(87,5 \%)$ dos casos. O grupo G2 apresentou predominância $(62,5 \%)$, apresentando diferença estatística significante entre os grupos $(\mathrm{p}=0,02)$. Na posição do parto, apresentando predominância nos dois, sendo tanto $(100,0 \%)$ para o G1 e $(75,0 \%) \mathrm{G} 2$, não apresentando diferença entre os grupos $(0,319 \%)$, a laceração é mais visível no grupo $\mathrm{G} 1$ com $(37,5 \%)$ de $2^{\circ}$ grau e $3^{\circ}$ grau, apresentando diferença estatística significante entre os grupos $(\mathrm{p}=0,034)$, a sutura é mais presente em mulheres do grupo 1 , que em mulheres do grupo dois, com $(87,5 \%)$ e $(25,0 \%)$, respectivamente, estando correlacionado estatisticamente com $(\mathrm{p}=0,02)$.

Outro fator que influência da realização de episiotomia, é a ocorrência de complicação durante o parto $(\mathrm{p}=0,013)$. Enquanto $(62,5 \%)$ das mulheres que foram submetidas ao procedimento tiveram algum tipo de complicação durante o parto, enquanto todas as mulheres do grupo 2 não passaram por complicação durante o parto (Tabela 2).

Tabela 2. Características obstétricas da amostra. Vitoria da Conquista - BA, 2018

\begin{tabular}{|c|c|c|c|}
\hline Características & $\mathbf{G 1}^{1}(\mathrm{n}=8)$ & $\mathbf{G 2}^{2}(\mathrm{n}=8)$ & $p^{*}$ \\
\hline \multicolumn{4}{|l|}{ Tipo de parto, $n(\%)$} \\
\hline Vaginal & $7(87,5)$ & $2(25,0)$ & \multirow{3}{*}{0,02} \\
\hline Cesário & - & $5(62,5)$ & \\
\hline 1 vaginal e 1 cesário & $1(12,5)$ & $1(12,5)$ & \\
\hline \multicolumn{4}{|l|}{ Posição do parto, $n(\%)$} \\
\hline Deitada de costas & $8(100,0)$ & $6(75,0)$ & \multirow{3}{*}{0,319} \\
\hline Deitada de lado & - & $1(12,5)$ & \\
\hline Sentada/reclinada & - & $1(12,5)$ & \\
\hline \multicolumn{4}{|c|}{ Ocorrência de laceração vaginal/perineal, $n(\%)$} \\
\hline Não & $2(25,0)$ & $7(87,5)$ & \multirow{3}{*}{0,034} \\
\hline $2^{\circ}$ grau & $3(37,5)$ & $1(12,5)$ & \\
\hline $3^{\circ}$ grau & $3(37,5)$ & - & \\
\hline \multicolumn{4}{|c|}{$\begin{array}{l}\text { Registro de sutura vaginal/perineal ou cicatriz } \\
\text { de episiotomia, } n(\%)\end{array}$} \\
\hline Sim & $7(87,5)$ & $2(25,0)$ & \multirow{2}{*}{0,02} \\
\hline Não & $1(12,5)$ & $6(75,0)$ & \\
\hline \multicolumn{4}{|c|}{ Complicação durante o trabalho de parto ou o parto, $n(\%)$} \\
\hline Sim & $5(62,5)$ & - & \multirow{2}{*}{0,013} \\
\hline Não & $3(37,5)$ & $8(100,0)$ & \\
\hline
\end{tabular}

A incontinência urinária foi verificada com o uso do questionário ICIQ-SF. Embora a maioria $5(62,5 \%)$ das mulheres que realizaram episiotomia foram diagnosticadas com incontinência urinária, existem também casos frequentes da IU nas mulheres que não passaram 
pelo procedimento, assim a prevalência da IU não possui correlação estatística significante entre mulheres com episiotomia e não epsiotomia $(p=0,5)$ (Tabela 3 ).

A frequência com que perde urina, bem como a quantidade perdida e o impacto do problema no cotidiano, não estão correlacionados de forma significante com os casos de episiotomia, $(\mathrm{p}=0,482 ; \mathrm{p}=0,856$ e $\mathrm{p}=0,394$, respectivamente $)$.

Tabela 3. Características da incontinência urinária da amostra. Vitoria da Conquista - BA, 2018.

\begin{tabular}{|c|c|c|c|}
\hline Características & $\mathbf{G 1}^{\mathbf{1}}(\mathrm{n}=8)$ & $\mathbf{G 2}^{2}(\mathrm{n}=8)$ & $p^{*}$ \\
\hline \multicolumn{4}{|l|}{ Presença de incontinência, $n(\%)$} \\
\hline Continentes & $3(37,5)$ & $4(50,0)$ & \multirow{2}{*}{0,5} \\
\hline Incontinentes & $5(62,5)$ & $4(50,0)$ & \\
\hline \multicolumn{4}{|c|}{ Com que frequência perde urina, $n(\%)$} \\
\hline Nunca & $3(37,5)$ & $4(50,0)$ & \multirow{5}{*}{0,482} \\
\hline Uma vez por semana ou menos & $2(25,0)$ & $1(12,5)$ & \\
\hline Duas ou três vezes por semana & - & $1(12,5)$ & \\
\hline Uma vez ao dia & - & $1(12,5)$ & \\
\hline Diversas vezes ao dia & $3(37,5)$ & $1(12,5)$ & \\
\hline \multicolumn{4}{|c|}{ Quantidade de urina que perde, $n(\%)$} \\
\hline Nunca & $5(62,5)$ & $4(50,0)$ & \multirow{3}{*}{0,856} \\
\hline Uma pequena quantidade & $2(25,0)$ & $3(37,5)$ & \\
\hline Uma moderada quantidade & $1(12,5)$ & $1(12,5)$ & \\
\hline \multicolumn{4}{|l|}{ Impacto da $\mathbf{I U}^{3}$ no cotidiano, $n(\%)$} \\
\hline Nada & $3(37,5)$ & $4(57,1)$ & \multirow{4}{*}{0,394} \\
\hline Leve & $2(25,0)$ & - & \\
\hline Moderado & - & $1(14,3)$ & \\
\hline Grave & $3(37,5)$ & $2(28,6)$ & \\
\hline
\end{tabular}

${ }^{1}$ Grupo Episiotomia ; ${ }^{2}$ Grupo Ausência de Episiotomia; ${ }^{3}$ Incontinência Urinária; * Teste do Quiquadrado de Pearson; Fonte: Dados da pesquisa.

O vaginismo é uma disfunção sexual, onde se analisou a função sexual dessas mulheres. O FSFI vem para mostrar a função sexual das mulheres. Ele avalia a disfunção sexual por meio de 6 domínios que são: Desejo, excitação, lubrificação, orgasmo, satisfação e dor. (Tabela 4). 
Tabela 4. Função sexual da amostra. Vitória da Conquista BA, 2018.

\begin{tabular}{llccc}
\hline & G1 $^{2}(\mathrm{n}=8)$ & G2 $^{3}(\mathrm{n}=8)$ & \\
\hline Função Sexual & $\begin{array}{c}\text { Média } \pm \text { Desvio } \\
\text { padrão }\end{array}$ & Média \pm Desvio padrão & $p^{*}$ \\
\hline FSFI $^{I}$ & Desejo & $3,45 \pm 1,23$ & $4,12 \pm 1,55$ & 0,352 \\
& Excitação & $3,60 \pm 1,72$ & $4,46 \pm 1,23$ & 0,010 \\
& Lubrificação & $3,86 \pm 1,78$ & $4,38 \pm 1,07$ & 0,488 \\
Orgasmo & $3,45 \pm 1,78$ & $4,40 \pm 0,97$ & 0,209 \\
Satisfação & $4,0 \pm 2,0$ & $4,65 \pm 1,22$ & 0,02 \\
Dor & $2,60 \pm 1,35$ & $5,40 \pm 0,80$ & 0,041 \\
Média geral do instrumento & $22,51 \pm 8,64$ & $27,42 \pm 5,61$ & 0,031 \\
\hline
\end{tabular}

${ }^{1}$ Female Sexual Function Index; ${ }^{2}$ Grupo Episiotomia ; ${ }^{3}$ Grupo Ausência de Episiotomia; Teste t-Student Independente; Fonte: Dados da pesquisa.

Nos resultados os domínios variaram de 1,2 a 6,0. A média geral do instrumento permite mostrar se existe disfunção sexual (média abaixo de 26) ou não (média acima de 26).

Sendo escalas crescentes [todos os domínios fora DOR], ou seja, quanto maior a pontuação, melhor. A escala da dor foi invertida no próprio questionário.

Observou-se que em todos os domínios, o grupo ausência de episiotomia apresenta melhores resultados que as mulheres do outro grupo.

Em ambos os grupos existe disfunção sexual no domínio desejo, com média que estão abaixo do ponto de corte para esse domínio, que é 4,58. Não foi verificada diferença estatística significante para o domínio desejo entre os grupos $(\mathrm{p}=0,352)$.

Para os domínios Lubrificação e orgasmos, existe disfunção sexual apenas para as mulheres que realizaram a episiotomia, $(\mathrm{p}=0,488, \mathrm{p}=0,209$, respectivamente), porém, este resultado não apresentou correlação estatística significante.

Mulheres que realizaram a epsiotomia foram classificadas de acordo o FSFI com disfunção sexual nos domínios: Excitação, Satisfação e Dor. As médias desses domínios são sempre inferiores às médias das mulheres que não passaram pelo procedimento. Para esses domínios foi verificada diferença estatística significante entre os grupos.

O domínio DOR é bem interessante analisar, uma vez que a média deu bastante baixa $(2,60 \pm 1,35)$ para as mulheres que passaram pela episiotomia.

As mulheres do grupo 1 foram classificadas com presença de disfunção sexual geral, pois apresentaram a média geral do instrumento abaixo de 26 . O que não ocorreu com as mulheres do grupo 2, com média geral de 27,45 \pm 5,61. Com a correlação estatística significante, que é $\mathrm{p}=0,031$, infere-se que mulheres que fizeram a epsiotomia possuem disfunção sexual. 
Diante dos resultados, concluímos que a intervenção com epsiotomia afeta diretamente a função sexual dessas mulheres, principalmente no domínio DOR. Porém, não foi verificada influência na incontinência urinária. Concluímos também que é realizada em mulheres de idade maior e que as características obstétricas estão diretamente ligadas com a realização do procedimento.

\section{Discussão}

No estudo, foi realizada uma comparação da presença de vaginismo em mulheres submetidas à episiotomia, dividido entre dois grupos: as que sofreram episiotomia e as que não sofreram. Os resultados da análise dos aspectos demográficos e clínicos encontrados neste estudo demonstraram que os dois grupos avaliados não são estatisticamente semelhantes em relação a estas variáveis, pois, o p-valor é menor que 0,05 .

A mulher que apresenta tais complicações causadas pela episiotomia possui maior risco de trauma perineal posterior, complicações na cicatrização, risco de infecção, dispareunia e dor perineal. A vida sexual das mulheres que são submetidas à episiotomia é afetada basicamente nos primeiros meses após o procedimento (PINHEIRO, 2012).

A Organização Mundial de Saúde (OMS) oferece uma taxa ideal de episiotomia nos diversos serviços em torno de $10 \%$, realidade em muitos países europeus, mas, infelizmente, no Brasil, em alguns centros, a situação é ainda adversa, pois o procedimento é realizado em, aproximadamente $94 \%$ dos partos vaginais. E, o presente estudo vem em consonância a essa afirmativa, ao encontrar que das 16 mulheres que tiveram filhos de parto vaginal 8 delas no G1, tiveram episiotomia resultando em 50\% da nossa amostra (PEREIRA, 2015).

Ao estudar dispareunia, dor perineal e cicatrização pós episiotomia verificou dor perineal em $16,5 \%$, e no presente estudo obteve a média bastante baixa de $(2,60 \pm 1,35)$ para o quesito dor, concluindo que a dor perineal e a dispareunia são morbidades constantes no pósparto com episiotomia (SILVA et al., 2013).

Encontrou na amostra de mulheres pesquisadas que 43,5\% apresentaram disfunções sexuais informando que os tipos de disfunção detectados com maior repetição foram dispareunia, seguida do vaginismo, disfunção do desejo, orgástica e excitação 4,58\%. Diante disso, não foi verificada diferença estatística significante entre os grupos deste estudo em relação ao desejo, lubrificação e orgasmo ( $p=0,352$ ) (FAUSTINO; ROVINSKI; BINI, 2017). 
O uso da episiotomia ainda permanece acontecendo de forma indiscriminada não atendendo às várias evidências de que o seu uso deve-se restringir a situações clínicas específicas uma vez que muitas são as complicações associadas ao uso rotineiro, como a incontinência urinária e as disfunções sexuais, as quais também puderam ser evidenciadas neste presente estudo, visto que $50 \%$ da população estudada foi submetida à episiotomia (TABISEL, 2002).

Concluindo que há necessidade de intervenção educacional para programar mudança dessa prática, uma vez que a utilização da episiotomia deve ser indicada para reduzir o período expulsivo e prevenir cruéis danos perineais em mulheres submetidas ao parto normal, diminuindo a ocorrência de toco traumatismos.

Esse trabalho teve como limitações o local da aplicação do instrumento de avaliação, ou seja, o número da amostra foi pequeno devido à frequência de poucos pacientes que se enquadravam no estudo. Dessa forma, se faz necessário que novas pesquisas para comprovação e esclarecimento desta temática sejam realizadas, com uma amostra maior e um período de pesquisa mais estendido.

\section{Conclusão}

Diante disso, o presente estudo mostra que houve presença dos sintomas do vaginismo em mulheres que foram submetidas à episiotomia, afetando diretamente na função sexual. Essa pesquisa é importante para conhecer os sintomas apresentados pelas mulheres submetidas à episiotomia, e a influência do vaginismo para a sua qualidade de vida. Diante deste contexto, torna-se importante a realização deste estudo, uma vez que a presença do vaginismo em mulheres que foram submetidas à episiotomia, afeta diretamente na qualidade de vida dessas mulheres.

Conclui-se, também que a maioria das mulheres que realizaram e as que não realizaram a episiotomia apresentam incontinência urinária, influenciando especialmente no impacto do cotidiano, e que deve ser feito mais pesquisas relacionadas ao tema, devido a grande importância do conhecimento para a população, uma vez que existem poucos estudos que demonstrem a relação destes sintomas com o procedimento.

Dessa forma, salienta-se a importância da educação continuada dos profissionais de saúde, permitindo novas discussões sobre as técnicas intervencionistas utilizadas na medicina 
moderna e suas implicações na vida do ser humano proporcionando, assim, uma assistência mais humanizada.

\section{Referência}

CAMBOIM, Francisca Elidivânia de Farias et al. História oral de vida temática de mulheres em relação à episiotomia. Arquivos de Ciências da Saúde, v. 24, n. 2, p. 25-32, 2017.

DE LIMA, Marcia Guerino et al. A Episiotomia e o retorno à vida sexual pós-parto. Revista UNINGÁ Review, v. 16, n. 2, 2018.

FAUSTINO, Elizabete C.; ROVINSKI, Edivania; BINI, Isabel. Atuação Fisioterapêutica Na Vulvodínia e Vaginismo. Vitrine de Produção Acadêmica Produção de Alunos da Faculdade Dom Bosco, v. 3, n. 2, 2017.

HOLANDA, Juliana Bento de Lima, et al. Disfunção sexual e fatores associados relatados no período pós parto. Acta Paul Enferm 2014.

PEREIRA, Amanda Cristina Gerhardt. Prevalência das disfunções do assoalho pélvico em mulheres com episiotomia. 2015. Disponível em <_http://hdl.handle.net/123456789/1552>. Acesso em 18/10/2018.

PINHEIRO, C. Parto Vaginal e Disfunções do Pavimento Pélvico - Novas Perspectivas. Faculdade de Medicina Universidade do Porto, março. 2012.

TABISEL, Ross Lynn. Private Pain-It's About Life, Not Just Sex: Understanding Vaginismus and Dyspareunia. Book Baby, 2002.

SILVA, Nathália Luiza Souza et al. Dispareunia, dor perineal e cicatrização após episiotomia. Rev. enferm. UERJ, v. 21, n. 2, p. 216-220, 2013.

Como citar este artigo (Formato ABNT):

ALVES, Andressa Moura; CIRQUEIRA, Rosana Porto. Sintomas do Vaginismo em Mulheres Submetidas à Episiotomia. Id on Line Rev.Mult. Psic., 2019, vol.13, n.43, p. 329-339. ISSN: 1981-1179.

Recebido: 25/11/2018;

Aceito: $26 / 11 / 2018$ 


\section{QUESTIONÁRIOS}

\section{FICHA DE AVALIAÇÃO OBSTÉTRICA}

\section{ANAMNESE: \\ IDENIFICAÇÃO:}

Data da coleta: Telefone

Nome:

\section{Endereco:}

Idade: Sexo: ( )Masculino ( )Feminino. Data de Nascimento:

Estado Civil: ( )Solteiro ( )Casado ( )Divorciado ( )Viúvo. Cor: ( ) Branca ( ) Amarela ( ) Parda Negro.

Profissão:

Escolaridade: ( )Analfabeto ( )Fundamental completo ( )Incompleto ( )Ensino médio incompleto ( )Ensino médio completo ( )Nível superior completo ( ) incompleto. Tabagismo: ( )fumante ( )ex fumante ( )não fumante. Obesidade: ( )Sim ( )Não. Sedentarismo: ( )Sim ( )Não

Qual sintoma apresenta: ( )dor ( )ardência ( )desconforto ( )incapacidade de ter relação sexual

Tipo de parto: ( )Vaginal ( )Cesáreo

Posição no parto: ( )Deitada de costas ( )Deitada de lado ( ) De pé ( )Sentada/reclinada ( )Na banheira ( )De quatro ( )De cócoras Realização de episiotomia: ( )Sim ( )Não

Ocorrência de laceração vaginal/perineal: ( )Não ( ) $1^{\circ}$ grau ( ) $2^{\circ}$ grau ( ) $3^{\circ}$ grau ( ) $4^{\circ}$.grau ( )Sim, sem especificação

Registro de sutura vaginal/perineal ou cicatriz de episiotomia: ( )Sim ( )Não Houve alguma complicação durante o trabalho de parto ou o parto?: ( )Sim ( )Não 


\section{ICIQ-SF}

Nome do Paciente:

Data de Hoje:

Muitas pessoss perdem urina alguma vez. Estamos tentando descobrir quantas pessoss perdem urine $e \circ$ quanto isso as aborrece. Ficarlamos agredecidos se você pudesse nos responder às seguintes perguntas, pensando em como você lem passado, em média nas ULLTIMAS QUATRO SEMANAS.

1. Data de Nascimento;

2. Sexo: Feminino $\square$ Masculino $\square$

( Dia / Más / Ano)

3. Com que frequéáciavoce perde urina? (assinale uma resposta)

Nunca

Uma vez por semana ou menos

Duas ou trés vezes por semana

Uma vez ao dia

Diversas vezes ao dia

O tempo todo

4. Gostariamos de saber a quantidade de urina que vocé pensa que perde (assinale uma resposta)

Nenhume

Uma pequena quanticade

Uma moderada quantidade

Uma grande quantidade

5. Em geral quanto que perder urina intertere em sua vida diária? Por favor, circule um número entre 0 (näo interfere) e 10 (interfere muito)

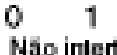
2
4
56
$7 \quad 8 \underset{\text { Interfere muito }}{9}$

ICIQ Escore: soma dos resultados $3+4+5=$

6. Quando você perde urina?

(Por favor assinale todas as alternativas que se aplicam a vocē)

Nunce

Perco antes de chegar ao banheiro

Pereo quando tusso ou espiro

Perco quando estou dormindo

Perco quando estou fazendo atividades fisicas

Perco quando terminei de urinar $\theta$ estou me vestindo

Perco sem razĩo óbvia

Perco o tempo todo

"Obrigado por você ter respondido às questōes"

Figura - Versão em português do ICIQ-SF. 
NSTHUÇOES

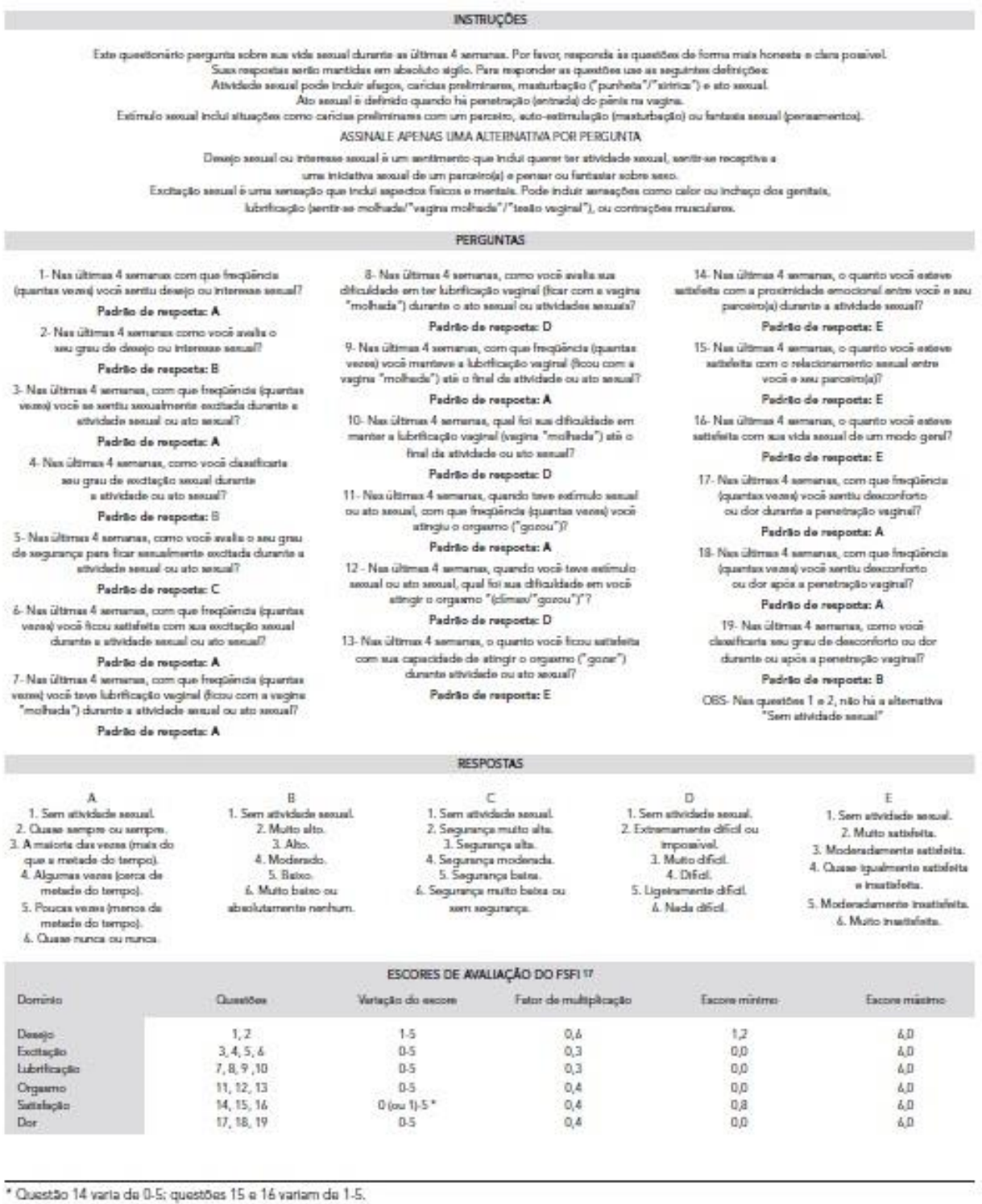

